Gefässchirurgie $2020 \cdot 25: 217-218$

https://doi.org/10.1007/s00772-020-00656-x

(c) Springer Medizin Verlag $\mathrm{GmbH}$, ein Teil von Springer Nature 2020

\author{
A. Gombert ${ }^{1,2} \cdot$ M. Hakimi ${ }^{2,3}$ \\ ' Klinik für Gefäßchirurgie, Europäisches Gefäßzentrum Aachen-Maastricht, Universitätsklinikum RWTH \\ Aachen, Aachen, Deutschland \\ ${ }^{2}$ Kommission für Wissenschaft und Forschung, Deutsche Gesellschaft für Gefäßchirurgie und \\ Gefäßmedizin, Berlin, Deutschland \\ ${ }^{3}$ Klinik für Gefässchirurgie, Luzerner Kantonsspital, Luzern, Schweiz
}

\title{
Grundlagenforschung durch Gefäßchirurgen - ein gutes, ein weites Feld
}

Liebe Leserinnen, liebe Leser, sehr geehrte Kolleginnen und Kollegen,

mit einem Augenzwinkern bedienen wir uns der berühmten Phrase von Theodor Fontane aus seinem bedeutenden Roman „Effi Briest“. Mitnichten möchten wir hier jedoch etwas Unangenehmes ausweichend beschreiben, sondern vielmehr möchten wir betonen, wie vielfältig und aussichtsreich die in diesem Heft vorgestellte Forschung ist.

Ohne den Anspruch zu haben, die gesamte Forschungslandschaft zu repräsentieren, möchte diese Sonderausgabe zweierlei:

Zum einen sollen die vielfältigen Forschungsansätze der Mitglieder unserer Fachgesellschaften vorgestellt werden.

Die hier vorgestellten Schwerpunkte reichen von zellulärer Biologie, konkret dem veränderten Mitochondrienstoffwechsel bei peripherer Minderperfusion, über die Beschreibung verschiedener Klein- und Großtiermodelle in der Atheroskleroseforschung. In einer Übersichtsarbeit wird die seltene Entität der multifokalen Aneurysmen beleuchtet, zudem werden biomechanische Untersuchungen, wie die Mechanotransduktion der Aorta, sowie Stoffwechselprodukte wie vaskuläre Lipide und perioperative Biomarker in den hervorragenden Arbeiten der forschenden Gefäßchirurgen und ihrer Arbeitsgemeinschaften vorgestellt.

Zum anderen soll, und dieser Aspekt liegt uns besonders am Herzen, der wissenschaftliche Dialog zwischen den Standorten, Regionen und Ländern gestärkt werden. Der Stellenwert der Grundlagenforschung kann in den Augen der Fachgesellschaften nicht genug betont werden, wie im Rahmen der Dreiländertagung 2019 in Mannheim mehrfach betont wurde. Die Grundlagenforschung durch Gefäßchirurgen muss im Spannungsfeld zwischen klinischer Tätigkeit und sich verknappenden Ressourcen existieren und wird zudem noch durch zu wenige, hoch motivierte Kollegen getragen. Die Chancen einer gut vernetzten und offen kommunizierenden Fachgesellschaft liegen darin, die Expertise einzelner Standorte zu verknüpfen: Durch Kooperationen, Hilfestellungen und Beratung bei wissenschaftlichen Projekten. In Ihrem letzten Treffen hat die Kommission für Wissenschaft und Forschung der DGG entsprechend beschlossen, diese Art des Austausches und der Zusammenarbeit fokussiert zu unterstützen, um Erfolg versprechende Bedingungen für forschende Gefäßchirurgen $\mathrm{zu}$ fördern.

\section{》) Der Stellenwert der Grundlagenforschung kann nicht genug betont werden}

Ein wichtiger Aspekt dabei ist die Wahrnehmung dieses wichtigen Beitrags für unser Fach und der angeregte Austausch mit den mehrheitlich rein klinisch ausgerichteten Kollegen. Mit dem Ansinnen, 
eine lebhafte und motivierende Atmosphäre für translationale Diskussionen zu schaffen, wurde die Gestaltung dieser Ausgabe der Zeitschrift Gefässchirurgie konzipiert. Der Diskurs zwischen Grundlagenforschung und klinischen Fragestellungen ist unabdingbar und sollte ein inspirierendes Element unserer Fachgesellschaften darstellen.

Wir hoffen, dass auch Sie, geschätzte Leserinnen und Leser, Anregung für Ihre eigene klinische und/oder wissenschaftliche Tätigkeit aus dieser Ausgabe der Gefässchirurgie ziehen. Wir möchten uns an dieser Stelle bei allen beteiligten Autoren für die hochwertigen und sehr gelungenen Beiträge und die hervorragende Zusammenarbeit bedanken.

Mit kollegialen Grüßen,

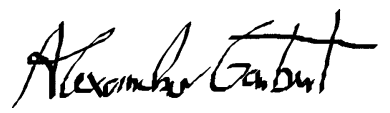

PD Dr. Alexander Gombert

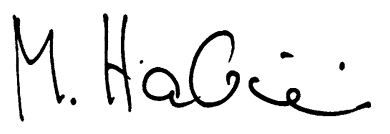

Prof. Dr. Maani Hakimi

\section{Korrespondenzadresse}

PD Dr. A. Gombert
Klinik für Gefäßchirurgie,
Europäisches Gefäßzentrum
Aachen-Maastricht,
Universitätsklinikum RWTH
Aachen
Pauwelsstraße 30,
52074 Aachen, Deutschland
agombert@ukaachen.de

Interessenkonflikt. A. Gombert und M. Hakimi geben an, dass kein Interessenkonflikt besteht.

Q SpringerMedizin

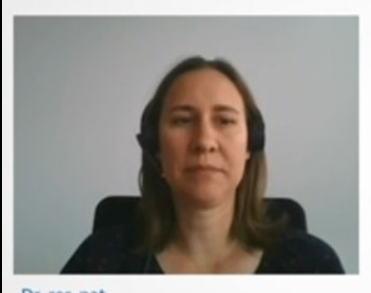

Dr.rer, nat

Tina Suhai

Springer Medizin Verlag GmbH

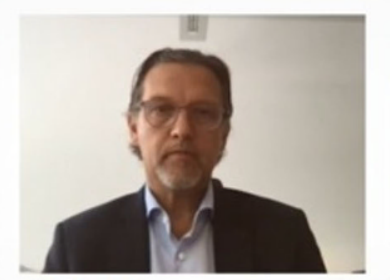

Prof. Dr. med.

Dittmar Böckler

Ärztlicher Direktor der Klinik für GefäBchirurgie und Endovaskulăre Chirurgie am

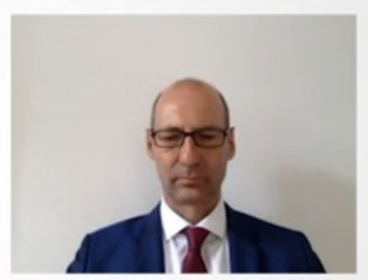

Prof. Dr. med.

Markus Steinbauer Leiter des Gefäzentrums am Krankenhaus Barmherzige Brüder Regensburg

\section{Corona-Webinare bei Springer Medizin}

\section{Webinare und Videointerviews über das Ärzteportal SpringerMedizin.de}

Springer Medizin hat seit April die Initiative Corona-Webinar über sein Ärzteportal (www.springermedizin.de) gestartet. Ärzte, die auf diesem Portal registriert sind, gelangen über Videoseminare an kostenloses Wissen rund um das Corona-Virus. In jedem Webinar oder Videointerview kommt ein Experte zu einem bestimmten Thema zu Wort und gibt fachlich gesichertes Wissen an Kollegen weiter, die sich mit Symptomatik, Diagnostik und Therapie in Klinik und Praxis beschäftigen. Wöchentlich kommen neue Themen hinzu!

In diesem Rahmen stehen seit Kurzem auch zwei Webinare zu den Themen „Erhöhte Thrombose- und Embolierate bei COVID-Patienten? Was wissen wir aktuell?" von Herrn Prof. Dr. M. Steinbauer (Regensburg) und "Wie kann sich medizinisches Personal vor einer Ansteckung schützen?" von Herrn Prof. Dr. D. Böckler (Heidelberg) zur Verfügung.

Auf der zentralen Corona-Update-Seite auf SpringerMedizin.de sind unter anderem bereits folgende Webinare verfügbar:

- Der Corona-Patient zwischen Hausarztpraxis und Corona-Ambulanz

- Kardiologische Implikationen und Komplikationen von COVID-19

- COVID-19: Symptomatik bei Säuglingen, Kindern, Jugendlichen

- Impfstoffe und Therapien gegen SARSCoV-2: Was kann welcher Kandidat?

- Infektiologe über SARS-CoV-2: "Wir werden dieses Virus nicht ewig bei uns haben"

- COVID-19-Pandemie: Aktuelles zu Epidemiologie, Diagnostik und
Therapiestrategien

- Covid-19 in der Klinik: "Fast Progressors", klinische Charakteristika und prognostische Marker

Alle Webinare sind über www.springermedizin.de/covid-19 zugänglich, die Seite wird ständig aktualisiert und erweitert.

„In unserer Ärztekommunikation werden wir das Webinar-Format zukünftig stärker nutzen, denn es ist eine sehr wertvolle Ergänzung zu den bestehenden Print- und OnlineAngeboten", erläutert Cécile Mack, Director Digital Product \& Marketing von Springer Medizin. „Die Inhalte eines Webinars oder VideoInterviews können durch die direkte Ansprache schneller aufgenommen werden. Unsere Redakteure der verschiedenen Magazine und Zeitschriften laden zum Ausbau dieser Initiative ausgewählte Autoren und Herausgeber ein, ihr Fachwissen zu Corona zu teilen. Neben den aktuellen Aspekten werden wir auch in den nächsten Monaten das Thema eng begleiten und über die Erkenntnisse aus klinischen Studien sowie der Entwicklung von Therapieoptionen und Impfstoffen berichten. Solange die SARS-CoV-2/Covid-19-Pandemie eine Bedrohung unserer Gesellschaft darstellt, wird Springer Medizin als ein führender Marktvertreter diese Inhalte kostenfrei vor der Bezahlschranke platzieren, um allen Ärzten eine sichere und fundierte Wissensbasis zu geben. Denkbar ist außerdem, dieses Format auch auf andere medizinische Themen oder zusätzliche Fachgebiete auszudehnen", so die Digitaldirektorin. 